\title{
Geometric methods for constructing cosmological solutions for EYMH systems in two measure, $f(R)$ and massive gravity theories
}

\author{
Subhash Rajpoot \\ California State University at Long Beach; Long Beach, California, USA \\ E-mail: Subhash.Rajpoot@csulb.edu
}

Sergiu I. Vacaru

Rector's Department, Alexandru Ioan Cuza University, UAIC

Al. Lapuşneanu str., nr. 14, UAIC - Corpus R, office 323; Iaşi, Romania, 700057; and* M. Planck Inst. Physics (W. Heisenberg Inst.), Föhringer Ring 6, München D-80805, Germany; Leibniz Univ. Hannover, Instit. Theoretical Physics, Appelstrasse 2, Hannover 30167, Germany E-mail: sergiu.vacaru@uaic.ro; Sergiu.Vacaru@gmail.com

\begin{abstract}
A brief summary of the anholonomic frame deformation method, AFDM, for generating exact solutions with generic off-diagonal metrics and generalized nonlinear connections in modified gravity theories, MGTs, is presented. We generalize the method to systems of nonlinear partial differential equations, PDEs, for constructing solutions describing generalized (effective) Einstein-Yang-Mills-Higgs, EYMH, interactions in two measure, $\mathrm{f}(\mathrm{R})$ and massive gravity theories. Finally, we speculate on possible applications of such generalized anisotropic and inhomogeneous solutions in modern acceleration cosmology.

Keywords: Geometric methods in modified gravity theories and cosmology; two measure theories; massive gravity; off-diagonal exact solutions.
\end{abstract}

\section{Nonholonomic variables \& Lagrangians for EYMH in MGTs}

We reexamine the issue of constructing and searching for physical implications of exact solutions depending generically on all spacetime coordinates ${ }^{1,2,6}$ in $f\left(\mathbf{R}, \mu_{0}\right)$ MGTs and when the corresponding (effective) Lagrangians determine two measure theories $^{3,7,8}$ (TMTs; in particular, bimetric and massive theories), and possible variants of Finsler like nonolonomic deformation on an effective spacetime with $2+2+\ldots$ splitting, or on a tangent Lorenz bundle ${ }^{2}$. Here $\mu_{0}$ is a mass parameter for gravity and $\mathbf{R}$ is the curvature scalar for a generalized connection. In these theories, the modified gravitational \& matter field eqs of TMTs are equivalent to certain effective EYMH systems, which can be solved using geometric methods.

The underlying principle of the $\mathrm{AFDM}^{1,2,5,6}$ is that we should re-write equivalently the Einstein equations, or their various MGTs modifications, on a (pseudo) Riemannian manifold $\mathbf{V}$ with nonholonomic splitting associated with a nonlinear connection (N-connection) structure $\mathbf{N}=\left\{N_{i}^{a}(u)\right\}$ for a conventional horizontal (h) and vertical (v) decomposition $\mathbf{N}: T \mathbf{V}=h \mathbf{V} \oplus v \mathbf{V}$, where $T \mathbf{V}$ is the tangent bundle $^{\dagger}$. All geometric and physical objects and equations should be written in

\footnotetext{
*DAAD fellowship affiliations for two host institutions

†Boldface and/or "hat" symbols will be used in order to emphasize that certain spaces and/or geometric objects are adapted to a $\mathrm{N}$-connection and satisfy corresponding properties. The metric splits $\mathbf{g}=\left\{\mathbf{g}_{\alpha \beta}=\left[g_{i j}, g_{a b}\right]\right\}$. If $\operatorname{dim} \mathbf{V}=4=2+2$, for signature $(+,+,+,-)$, then $i, j, k, \ldots=1,2$ while $a, b, \ldots=3,4$; and local coordinates are denoted by $u^{\alpha}=\left(x^{i}, y^{a}\right)$, or equivalently, $u=(x, y)$.
} 
in terms an "auxiliary" linear connection $\widehat{\mathbf{D}}=\nabla+\widehat{\mathbf{Z}}[\widehat{\mathbf{T}}]$ uniquely defined by the conditions

$$
\mathbf{g} \rightarrow \begin{cases}\nabla: \quad \nabla \mathbf{g}=0 ; \nabla^{\nabla} \mathbf{T}=0, \quad \text { the Levi-Civita connection; } \\ \widehat{\mathbf{D}}: \widehat{\mathbf{D}} \mathbf{g}=0 ; h \widehat{\mathbf{T}}=0, v \widehat{\mathbf{T}}=0, & \text { the canonical d-connection, }\end{cases}
$$

where $\nabla$ is the Levi-Civita (LC) connection.

The advantage of $\widehat{\mathbf{D}}$ is that in this framework certain "hatted" EYMH eqs

$$
\begin{aligned}
\widehat{\mathbf{R}}_{\alpha \beta}-\frac{1}{2} \widehat{\mathbf{g}}_{\alpha \beta} \widehat{R} & =\boldsymbol{\Upsilon}_{\alpha \beta}, \\
(\sqrt{|\widehat{\mathbf{g}}|})^{-1} D_{\mu}\left(\sqrt{|\widehat{\mathbf{g}}|} F^{\mu \nu}\right) & =\frac{1}{2} i e\left[\phi, D^{\nu} \phi\right],(\sqrt{|\widehat{\mathbf{g}}|})^{-1} D_{\mu}(\sqrt{|\widehat{\mathbf{g}}|} \phi)=\lambda\left(\phi_{[0]}^{2}-\phi^{2}\right) \phi,
\end{aligned}
$$

decouple with respect to $\mathrm{N}$-adapted frames for various classes of metrics and effective sources. This procedure allows to construct very general classes of generic off-diagonal solutions determined by corresponding data for generation and integration functions and constants ${ }^{1,2,5,6} \ddagger$ Here the hatted Ricci tensor $\widehat{\mathbf{R}}$ and (effective) source $\Upsilon$ are defined in standard form following geometric methods and $\mathrm{N}$-adapted variational calculus but for the data $(\mathbf{g}, \widehat{\mathbf{D}})$ instead of $(\mathbf{g}, \nabla)$, where the source $\left.\Upsilon_{\alpha \beta}(u)=\frac{\kappa}{2}\left({ }^{\phi} T_{\beta \delta}+{ }^{F} T_{\beta \delta}\right)\right)$ is determined by the stress-energy tensor

$$
\begin{aligned}
& { }^{\phi} T_{\beta \delta}=\operatorname{Tr}\left[\frac{1}{4}\left(D_{\delta} \phi D_{\beta} \phi+D_{\beta} \phi D_{\delta} \phi\right)-\frac{1}{4} \widehat{\mathbf{g}}_{\beta \delta} D_{\alpha} \phi D^{\alpha} \phi\right]-\widehat{\mathbf{g}}_{\beta \delta}{ }^{e} V(\phi), \\
& { }^{F} T_{\beta \delta}=2 \operatorname{Tr}\left(\widehat{\mathbf{g}}^{\mu \nu} \mathbf{F}_{\beta \mu} \mathbf{F}_{\delta \nu}-\frac{1}{4} \widehat{\mathbf{g}}_{\beta \delta} \mathbf{F}_{\mu \nu} \mathbf{F}^{\mu \nu}\right) .
\end{aligned}
$$

The nonlinear potential ${ }^{e} V(\phi)$ is taken for a TMT (see below). In the above formulae, we consider $\phi$ as a Higgs field (with interior indices and vacuum expectation $\left.\phi_{[0]}\right)$ coupled to the gauge field $\mathbf{A}=\mathbf{A}_{\mu} e^{\mu}$, in general, with values in non-Abelian Lie algebra. It defines a "proper" covariant derivative $D_{\mu}=\mathbf{e}_{\mu}+i e\left[\mathbf{A}_{\mu},\right]$ which is characterized by curvature $F_{\beta \mu}=\mathbf{e}_{\beta} \mathbf{A}_{\mu}-\mathbf{e}_{\mu} \mathbf{A}_{\beta}+i e\left[\mathbf{A}_{\beta}, \mathbf{A}_{\mu}\right]$.

The equations (1) can be derived from a TMT like action ${ }^{3,7}$ for equivalent modelling of massive MGT and nonholonomically deformed Einstein models,

$$
\begin{aligned}
\mathcal{S}= & \left({ }^{P l} M\right)^{2} \int d^{4} u \sqrt{|\widehat{\mathbf{g}}|}[\widehat{R}+\widehat{\mathcal{L}}]={ }^{\Phi} \mathcal{S}^{m}+\mathcal{S}=\int d^{4} u{ }^{1} \Phi(\mathbf{A})\left[\widehat{R}+{ }^{1} L\right] \\
& +\int d^{4} u^{2} \Phi(\mathbf{B})\left[{ }^{2} L+\epsilon \mathbf{f}(\widetilde{\mathbf{R}})+(\sqrt{|\mathbf{g}|})^{-1} \Phi(\mathbf{H})\right]+\int d^{4} u \sqrt{|\widehat{\mathbf{g}}|}^{m} \mathcal{L} \\
= & { }^{F, \mu} \mathcal{S}+{ }^{m} \mathcal{S}=\left({ }^{P l} M\right)^{2} \int d^{4} u\left[\sqrt{|\widehat{\mathbf{g}}|}{ }^{F, \mu} \mathcal{L}+\sqrt{|\widehat{\mathbf{g}}|}{ }^{m} \mathcal{L}\right],
\end{aligned}
$$

where $|\widehat{\mathbf{g}}|=\operatorname{det}\left|\widehat{\mathbf{g}}_{\alpha \beta}\right|$ and ${ }^{\Phi} L$ defines a class of theories with two independent nonRiemannian volume-forms ${ }^{1} \Phi(A)$ and ${ }^{2} \Phi(B)$ as in ${ }^{3,7}$ but with a more general functional for modification, of the type $\epsilon \mathbf{f}(\widetilde{\mathbf{R}})$, than $\epsilon R^{2}$ if $\widehat{\mathbf{D}} \rightarrow \nabla$. The Lagrange

$\ddagger$ It should be noted that $\widehat{\mathbf{D}}$ contains nontrivial anholonomically induced torsion $\widehat{\mathbf{T}}$ related to a nonholonomic frame structure and $\widehat{\mathbf{T}}$ can be taken to be zero after the relevant classes of solutions have been constructed in explicit form. Such a torsion field is completely defined by the metric and nonholonomic (equivalently, anholonomic and/or non-integrable) distortion relations. 
density functional ${ }^{f, \mu} \mathcal{L}=\mathbf{F}(\widetilde{\mathbf{R}})$ determined similar to a modified massive gravity by a mass-deformed scalar curvature ${ }^{4,6}$,

$$
\tilde{\mathbf{R}}:=\widehat{\mathbf{R}}+2 \mu^{2}\left(3-\operatorname{tr} \sqrt{\mathbf{g}^{-1} \mathbf{q}}-\operatorname{det} \sqrt{\mathbf{g}^{-1} \mathbf{q}}\right),
$$

where $\mu$ is the graviton's mass and $\mathbf{q}=\left\{\mathbf{q}_{\alpha \beta}\right\}$ is the so-called non-dynamical reference metric; ${ }^{m} \mathcal{L}$ is the Lagrangian for matter fields. There are also considered

$$
{ }^{1} L=-\frac{1}{\kappa} \widehat{R}(\mathbf{g})+\frac{1}{2} \mathbf{g}^{\mu \nu} \mathbf{e}_{\mu} \phi \mathbf{e}_{\nu} \phi-V(\phi) \text { and }{ }^{2} L=U(\phi)-\frac{1}{4} F_{\mu \nu}^{a} F^{a \mu \nu},
$$

for an effective source for a scalar field $\phi$, a gauge field $\mathbf{F}_{\mu \nu}^{a}$, which results in an effective nonlinear scalar potential ${ }^{e} V$ is determined by two scalar potentials $V(\phi)$ and $U(\phi)$ as ${ }^{e} V=(V+M)^{2} / 4 U$ and $M$ is a constant.

The action $S$ is constructed for the data $(\widehat{\mathbf{g}}, \widehat{\mathbf{D}})$, with a new 'scaled' metric $\mathbf{g}_{\alpha \beta}$, where $\widehat{\mathbf{g}}_{\alpha \beta}=e^{-2 \widehat{\sigma}(u)} \mathbf{g}_{\alpha \beta}$ and

$$
e^{-2 \widehat{\sigma}(u)}=2 U /(V+M)=\Phi / \sqrt{\left|\mathbf{g}_{\alpha \beta}\right|}
$$

is a scale factor $e^{-2 \widehat{\sigma}}$ determined by the constants and potentials used in the effective potential ${ }^{e} V$. The function

$$
\Phi=\varepsilon^{\mu \nu \alpha \beta} \mathbf{e}_{\mu} \mathbf{A}_{\nu \alpha \beta}=\varepsilon^{\mu \nu \alpha \beta} \varepsilon^{\frac{a b c d}{\mathbf{e}_{\mu}}} \varphi_{\underline{a}} \mathbf{e}_{\nu} \varphi_{\underline{b}} \mathbf{e}_{\alpha} \varphi_{\underline{c}} \mathbf{e}_{\beta} \varphi_{\underline{d}},
$$

with four scalar fields $\varphi_{\underline{a}},(\underline{a}=1,2,3,4)$, defines the second measure in TMTs.

\section{Off-diagonal Solutions for Effective EYMH Systems and Anisotropic Cosmological Attractors}

The ansatz for $\mathbf{F}_{\beta \mu}, A_{\mu}$ and $\phi$ and are nonholonomically constrained to the condition that ${ }^{F} T_{\beta}^{\alpha}=-4 s^{2} \delta_{\beta}^{\alpha}$, resulting in an effective cosmological constant ${ }^{s} \Lambda=8 \pi s^{2}$, when

$$
\mathbf{\Upsilon}_{\delta}^{\beta}=\operatorname{diag}\left[\mathbf{\Upsilon}_{\alpha}\right] \rightarrow \mathbf{\Upsilon}_{\delta}^{\beta}+{ }^{F} T_{\delta}^{\beta}=\operatorname{diag}\left[\mathbf{\Upsilon}_{\alpha}-4 s^{2} \delta_{\beta}^{\alpha}\right]
$$

We consider general off-diagonal ansatz for metrics depending on all spacetime coordinates,

$$
\begin{aligned}
\widehat{\mathbf{g}}= & e^{\psi\left(x^{k}\right)}\left[d x^{1} \otimes d x^{1}+d x^{2} \otimes d x^{2}\right]+ \\
& \omega^{2}\left(x^{k}, y^{3}, t\right)\left[h_{3}\left(x^{k}, t\right) \mathbf{e}^{3} \otimes \mathbf{e}^{3}+h_{4}\left(x^{k}, t\right) \mathbf{e}^{4} \otimes \mathbf{e}^{4}\right], \\
\mathbf{e}^{3}= & d y^{3}+n_{i}\left(x^{k}\right) d x^{i}, \mathbf{e}^{4}=d t+w_{i}\left(x^{k}, t\right) d x^{i},
\end{aligned}
$$


when the EYMH eqs (1) transform into such a system $\S$ of nonlinear PDEs,

$$
\begin{aligned}
\psi^{\bullet \bullet+\psi^{\prime \prime}}=2\left(\Upsilon-4 s^{2}\right), \partial_{t} \varpi \partial_{t} h_{3} & =2 h_{3} h_{4}\left(\Upsilon-4 s^{2}\right), \\
\partial_{t t}^{2} n_{i}+\gamma \partial_{t} n_{i}=0, \beta w_{i}-\alpha_{i} & =0, \\
\partial_{i} \omega-n_{i} \partial_{3} \omega-\left(\partial_{i} \varpi / \partial_{t} \varpi\right) \partial_{t} \omega & =0 .
\end{aligned}
$$

The solutions of this system are found integrating the eqs "step by step" and expressed in terms of an effective cosmological constant $\Lambda_{0}-4 s^{2} \neq 0$ and with generating functions with re-definition, ${ }^{s} \Psi \longleftrightarrow{ }^{s} \widetilde{\Psi}\left(x^{i}, t\right)$, when

$$
\begin{aligned}
{ }^{s} \Psi^{2} & =\left(\Lambda_{0}-4 s^{2}\right)^{-1} \int d t\left(\Upsilon-4 s^{2}\right) \partial_{t}\left({ }^{s} \widetilde{\Psi}^{2}\right), \\
{ }^{s} \widetilde{\Psi}^{2} d t & =\left(\Lambda_{0}-4 s^{2}\right) \int d t\left(\Upsilon-4 s^{2}\right){ }^{-1} \partial_{t}\left({ }^{s} \Psi^{2}\right) .
\end{aligned}
$$

The functional

$$
{ }^{s} \Xi\left[\Upsilon,{ }^{s} \widetilde{\Psi}\right]=\int d t\left(\Upsilon-4 s^{2}\right) \partial_{t}\left({ }^{s} \widetilde{\Psi}^{2}\right)
$$

can be considered as a re-defined source, $\Upsilon-4 s^{2} \rightarrow{ }^{s} \Xi$, for a prescribed generating function $\widetilde{\Psi}$, when $\Upsilon-4 s^{2}=\partial_{t}\left({ }^{s} \Xi\right) / \partial_{t}\left({ }^{s} \widetilde{\Psi}^{2}\right)$. Such effective sources contain information on effective off-diagonal EYMH, modified and massive gravity interactions in MGTs.

Summarizing the procedure of generating exact generic off-diagonal solutions of the system (2), we get a quadratic line element $d s^{2}=g_{\alpha \beta}\left(x^{k}, y^{3}, t\right) d u^{\alpha} d u^{\beta}$, where

$$
\begin{aligned}
d s^{2}= & e^{\psi(x, z)}\left[(d x)^{2}+(d z)^{2}\right]+\left({ }^{s} \omega\right)^{2} \frac{\left(\partial_{t} s \widetilde{\Psi}\right)^{2}}{s \Xi}\left[d t+\frac{\partial_{i}{ }^{s} \Xi}{\partial_{t}{ }^{s} \Xi} d x^{i}\right]^{2}, \\
& +\left({ }^{s} \omega\right)^{2} \frac{{ }^{s} \widetilde{\Psi}^{2}}{4\left(\Lambda_{0}-4 s^{2}\right)}\left[d y+\left({ }_{1} n_{k}(x, z)+{ }_{2} \widetilde{n}_{k}(x, z) \int d t \frac{\left(\partial_{4}{ }^{s} \widetilde{\Psi}\right)^{2}}{\left({ }^{s} \widetilde{\Psi}\right)^{3} s \Xi}\right) d x^{k}\right]^{2}
\end{aligned}
$$

where ${ }^{s} \omega(x, z, y, t)$ is a solution of

$$
\partial_{i} \omega-n_{i} \partial_{3} \omega-w_{i} \partial_{t} \omega=0 .
$$

The function $\psi(x, z)$ is a solution of

$$
\partial_{x x}^{2} \psi+\partial_{z z}^{2} \psi=2\left(\Upsilon-4 s^{2}\right)
$$

and the coefficients ${ }_{1} n_{k}(x, z)$ and ${ }_{2} \widetilde{n}_{k}(x, z)$ are integration functions.

Solutions (3) describe general anisotropic and inhomogeneous off-diagonal deformations of cosmological metrics in MGTs encoding modified EYMH interactions,

\footnotetext{
$\S$ The coefficients used are $\alpha_{i}=\left(\partial_{t} h_{3}\right)\left(\partial_{i} \varpi\right), \beta=\left(\partial_{t} h_{3}\right)\left(\partial_{t} \varpi\right), \gamma=\partial_{t}\left(\ln \left|h_{3}\right|^{3 / 2} /\left|h_{4}\right|\right)$, where $\varpi=\ln \left|\partial_{t} h_{3} / \sqrt{\left|h_{3} h_{4}\right|}\right|$ is a generating function; we suppose that $\partial_{t} h_{a} \neq 0$ and $\partial_{t} \varpi \neq 0$, (there are nontrivial solutions if such conditions are not satisfied but we have to consider more special methods for generating solutions); in brief, the partial derivatives $\partial_{\alpha}=\partial / \partial u^{\alpha}$ are labeled $\partial_{1} s=$ $s^{\bullet}=\partial s / \partial x^{1}, \partial_{2} s=s^{\prime}=\partial s / \partial x^{2}, \partial_{3} s=\partial s / \partial y^{3}, \partial_{4} s=\partial s / \partial t=\partial_{t} s, \partial^{2} s / \partial t^{2}=\partial_{t t}^{2} s$.
} 
massive gravity terms, two measure effects etc. ${ }^{1,3,5,7}$ Nonholonomic nonlinear interactions result in changing the topology and symmetries, include solitonic and black hole/ellipsoid and wormhole configurations, mimic dark energy and dark matter contributions $^{2,4,6}$. The most surprising thing is that using geometric methods, we can really integrate in very general forms the modified gravitational and matter field eqs in MGTs and parameterize such solutions via corresponding classes of generating and integration constants. For small nonholonomic off-diagonal deformations, such solutions have explicit physical meaning when the values of generating/integration functions and constants have to be chosen in some forms to describe observational effects in modern acceleration cosmology and astrophysics.

\section{Acknowledgments}

$\mathrm{SV}$ is supported by a travel grant from MG14 and reports certain research related to his basic activity at UAIC, a DAAD fellowship and the Program IDEI, PN-II-IDPCE-2011-3-0256. He is grateful for DAAD hosting to D. Lüst and O. Lechtenfeld.

\section{References}

1. S. Vacaru, JHEP 04, 009 (2001); arXiv: gr-qc/0005025.

2. S. Vacaru, Class. Quant. Grav. 27, 05003 (2010) ; arXiv: 0907.4278.

3. E. Guendelman, H. Nishino, S. Rajpoot, Phys. Rev. D 87, 027702 (2013).

4. S. Vacaru, EPJC 74, 2781 (2014); arXiv: 1403.1815.

5. S. Vacaru, E. Veliev, E. Yazici, IJGMMP 11, 1450088 (2014); arXiv: 1411.2849.

6. T. Gheorghiu, O. Vacaru, S. Vacaru, EPJC 74, 3152 (2014); arXiv: 1312.4844.

7. S. Rajpoot, S. Vacaru, Cosmological Attractors and Anisotropies in two Measure Theories, Effective EYMH systems, and Off-diagonal Inflation Models, under elaboration.

8. S. Vacaru, Phys. Lett. B 752, 27 (2016), arXiv: 1304.1080. 